\title{
Validation of the PHQ-15 for Somatoform Disorder in the Occupational Health Care Setting
}

\author{
Lars de Vroege $\cdot$ Rob Hoedeman · Jasper Nuyen • \\ Klaas Sijtsma $\cdot$ Christina M. van der Feltz-Cornelis
}

Published online: 22 July 2011

(c) The Author(s) 2011. This article is published with open access at Springerlink.com

\begin{abstract}
Introduction Within the occupational health setting, somatoform disorders are a frequent cause of sick leave. Few validated screening questionnaires for these disorders are available. The aim of this study is to validate the PHQ-15 in this setting. Methods In a cross-sectional study of 236 sicklisted employees, we studied the performance of the PHQ-15 in comparison with the Mini International Neuropsychiatric Interview (MINI) as golden reference standard. We approached employees who were sick listed for a period longer than 6 weeks and shorter than 2 years for participation. This study was conducted on one location of a large occupation health service in the Netherlands, serving companies with more than 500 employees.
\end{abstract}

L. de Vroege $(\varangle)$ · J. Nuyen · C. M. van der Feltz-Cornelis Trimbos Institute, Netherlands Institute of Mental Health and Addiction, Utrecht, The Netherlands

e-mail:1.vroege@trimbos.nl

R. Hoedeman

Department of Science, ArboNed Occupational Health Services, Utrecht, The Netherlands

R. Hoedeman

Department of Health Sciences, University Medical Center of Groningen, Groningen, The Netherlands

\section{K. Sijtsma}

Department of Methodology and Statistics,

Tilburg University, Tilburg, The Netherlands

C. M. van der Feltz-Cornelis

Department of Developmental and Clinical Psychology,

Tilburg University, Tilburg, The Netherlands

C. M. van der Feltz-Cornelis

Deptartment of Psychiatry, GGZ Breburg Groep,

Tilburg, The Netherlands
All employees who returned the PHQ-15 were invited for the MINI interview. Specificity and sensitivity were calculated for optimal cut point and a receiver operating characteristic (ROC) was constructed. Results A total of 107 participants consented to participate in the MINI interview. A non-response analysis showed no significant differences between groups. According to the MINI, the prevalence of somatoform disorders was $21.5 \%$, and the most frequent found disorder was a pain disorder. The PHQ-15 had an optimal cut point of 9 (patients scoring 9 or higher $(\geq 9)$ were most likely to suffer from a somatoform disorder), with specificity and sensitivity equal to 61.9 and $56.5 \%$, respectively. ROCs showed an area under the curve (AUC) of 0.63. Conclusion The PHQ-15 shows moderate sensitivity but limited efficiency with a cut point of 9 and can be a useful questionnaire in the occupational health setting.

Keywords PHQ-15 - Somatoform disorders . Validation study - Occupational health setting . Specificity $\cdot$ Sensitivity

\section{Introduction}

Medically Unexplained Symptoms (MUS) and somatoform disorders occur frequently in sicklisted employees at the workplace [1]. Somatoform disorders as defined as DSMIV category are established in this study by MINI interview. Frequently occurring somatoform disorders are pain disorders, which include pain with physical factors and pain with psychological factors. In this study, we use the term MUS for specific symptoms occurring in the context of somatoform disorders. In this study, MUS occur in combination with disfunctioning as they did lead to job 
disfunctioning resulting in the employees being sicklisted; this is an important prerequisite for classification as somatoform disorder.

Previous studies reported a prevalence of MUS in this setting ranging from 10 to $16 \%$ [1-3] and this often coincided with mental disorders such as depressive or anxiety disorders. Also, there are indications that somatoform disorders are often presented as musculoskeletal symptoms in this setting, inhibiting work functioning [2, 4-6]. In order to offer patients a suitable treatment, recognition is crucial. However, in the occupational health $(\mathrm{OH})$ setting, somatoform disorders are often not recognized [4]. A proper screening tool might be useful to improve recognition of somatoform disorders in the $\mathrm{OH}$ setting. The use of a routine screener such as the 15-symptom Patient Health Questionnaire (PHQ-15) could be very useful for the occupational health physician (OHP) to identify patients who require a more psychological approach. If a screener for somatoform disorder is used in an early phase, this might improve clinical decision-making and selection of relevant treatment options, even more so now that recently a multidisciplinary guideline for evidence-based treatment of somatoform disorders was published [7].

In this study, the Dutch version of the PHQ-15 was chosen for validation as a screener in the $\mathrm{OH}$ setting. The PHQ-15 is the somatic symptom severity scale of the Patient Health Questionnaire (PHQ), which is a short, self-report version of the Primary Care Evaluation of Mental Disorders (PRIME-MD) developed by Kroenke and colleagues [8]. The PHQ-15 consists of a list of 15 somatic symptoms. In a validation study in primary care in Germany, higher scores on the PHQ-15 were strongly associated with functional impairment, disability, and health care use [6]. In the Netherlands, in two studies in the $\mathrm{OH}$ setting in sick listed employees, higher scores on the PHQ-15 were associated with more disability, longer sickness absence and higher health-related job loss [3, 4]. In a recent review of studies in primary care, the PHQ-15 was found to be equally effective or superior to other brief measures for assessing somatic symptoms and screening for somatoform disorders, with cut points of 5, 10 and 15 representing mild, moderate and severe symptom levels. However, a validation of the PHQ-15 in the $\mathrm{OH}$ setting was not yet performed [9]. Van Ravesteijn et al. [9] validated the Dutch version of the PHQ-15 for the primary care setting. Therefore we expected the PHQ15 to be a valid instrument for the $\mathrm{OH}$ setting as well. The aim of this study was therefore to validate the PHQ15 in the $\mathrm{OH}$ setting by comparing the PHQ-15 with the MINI International Neuropsychiatric Interview (MINI; this is a short neuropsychiatric interview [10], see section 2.7) as golden standard.

\section{Method}

This validation study of the PHQ-15 was performed as part of a cross-sectional survey to assess the prevalence of severe MUS and psychiatric comorbidity in a sick-listed population [3], and to validate several questionnaires against the MINI-interview. Validation of the PHQ-15 is reported here. The Medical Ethics Committee approved of the study.

\section{Participants}

A total of 776 employees who were sick listed for a period longer than 6 weeks and shorter than 2 years were approached to participate at their visit of their OHP at a large occupation health services in the Netherlands (ArboNed), Corporate Accounts, from April 2006 until December 2007. Patients were recruited from one location of ArboNed, serving companies with more than 500 employees. Individuals unable to fill out the questionnaires (due to insufficient mastery of the Dutch language) and persons with psychotic symptoms or at increased risk for suicide, were excluded from the study. As procedure, over a period of 6 weeks 12 OHPs were asked to select a 4-h consultation session every week on the same day. The practice assistants in the administrative section of the $\mathrm{OH}$ service were instructed to invite all sick-listed employees, who had an appointment for this session to participate in the study. The employees who volunteered to participate received the research questionnaires (including the PHQ15) and an informed consent form 1 week before the actual consultation, or later if they received the invitation after that time. The OHPs were not involved themselves in the selection of the patients. The questionnaires were sent to the Trimbos Institute. After receipt, a blinded Trimbos Institute research assistant contacted the employee by telephone for the MINI interview. To assess the validity of the PHQ-15, we invited all participants who returned the PHQ-15 $(N=172)$ for a MINI interview within 2 weeks after receiving the PHQ-15. The interviewer was blinded for the scores of the participants on the questionnaires. This procedure is described more extensively elsewhere [3].

Assessment

Socio-demographic variables, depression, anxiety, distress, and MUS were assessed in this study.

\section{Objectives}

Primary objective was to validate the PHQ-15 for detecting somatoform disorders in the $\mathrm{OH}$ setting by using the MINI as golden standard. 
The PHQ-15

The PHQ-15 is the somatic subscale of the PHQ. It comprises almost all physical symptoms in the outpatient setting [11]. The PHQ-15 contains 15 items, 13 of which are based on a 3-point response scale, with ordered response categories 'not bothered at all' (0 points), 'bothered a little' ( 1 point) and 'bothered a lot' ( 2 points). The remaining 2 items consist of questions about 'feeling tired or having little energy' and 'trouble sleeping', which are contained in the depression module of the PHQ. Scores for these 2 questions can be $0,1,2$ or 3 points depending on the patient's response, which is either 'not at all', 'several days', 'more than half the days' or 'nearly every day'. Before adding these item scores to the sum of the total score based on the other 13 items in the PHQ-15, responses to these two questions were re-scored as described by Kroenke et al. ('not at all' with 0 points, 'several days' with 1 point and 'more than half of the days' or 'nearly every day' with 2 points) [11].

\section{MINI Interview}

The MINI interview was used as the golden standard in this study. Based on the DSM-IV criteria, Sheehan et al. [10] developed this interview, which is often used in the clinic. The MINI is used to diagnose and classify somatoform disorders. A trained, blinded research assistant of the Trimbos Institute conducted the MINI Interview by telephone. Participants were asked about physical symptoms within the previous 2 weeks to 6 months [10]. In case of any doubt whether or not a patient suffered from a medically explained or unexplained condition or pain symptom, an OHP (RH) or a psychiatrist (CFC) was to be consulted.

\section{Analysis}

\section{Construct Validity}

First, the mean PHQ-15 scores were calculated both for patients who according to the MINI suffered from somatoform disorders, and for subjects who did not. Also, demographic characteristics of both groups were recorded. Significance of differences was established by chi-square tests and $t$ tests. We expected that the PHQ-15 scores would differ between both groups.

\section{Diagnostic Validity}

For clinical diagnosis, a test needs to be sensitive enough to detect the relevant problem if it is present (and therefore avoid many false negative results), but specific enough to keep the number of false positives as low as possible.
Therefore, to assess the research question on the diagnostic validity of the PHQ-15, based on the sum score of the PHQ-15 the sensitivity, the specificity, and the predictive values were calculated for cut points ranging from 0 to 30 .

In order to discern whether the efficiency of the PHQ-15 exceeded randomness (50\%), sensitivity, specificity, predictive values, and efficiency (the total percentage of correct diagnosis, combining positive and negative diagnosis) were determined for different cut points. This way, an optimal cut point could be determined and a receiver operating characteristic (ROC) was calculated to explore diagnostic performance. An area under the curve (AUC) was also calculated. Analysis was performed using SPSS v15. As not all patients who returned the PHQ-15 subsequently consented to the MINI interview, a non-response analysis was performed.

\section{Results}

Flowchart

A flowchart of the study is provided in Fig. 1. 776 sicklisted employees were approached to fill out the PHQ-15, and $172(22.1 \%)$ returned the questionnaire. Eventually we analysed the data of 107 persons for whom we obtained both a PHQ-15 score and a MINI classification; this is $13.7 \%$ of the persons who were approached to participate for informed consent initially. In nine cases $(8 \%)$ the psychiatrist (CFC) was consulted regarding uncertainty about the patient suffering from pain syndrome or from medically explained pain without psychological factors. Among these nine cases, five were considered medically unexplained and were included as pain disorder; four were assigned to the 'no somatoform disorder'-group.

\section{Non-Response Analysis}

Of the 172 persons who received PHQ-15 questionnaires, 107 participants subsequently underwent the MINI interview, while 65 did not. PHQ-15 scores, demographic characteristics, gender, marital status, age and level of education did not differ significantly between responders and non-responders.

\section{MINI Classifications}

MINI classifications are shown in Table 1.

In the total sample $(N=107), 84$ participants did not fulfill diagnostic criteria and were not classified with a somatoform disorder according to the MINI interview (i.e. the "no-somatoform disorder group"), and 23 fulfilled classification criteria for somatoform disorders (i.e. the 


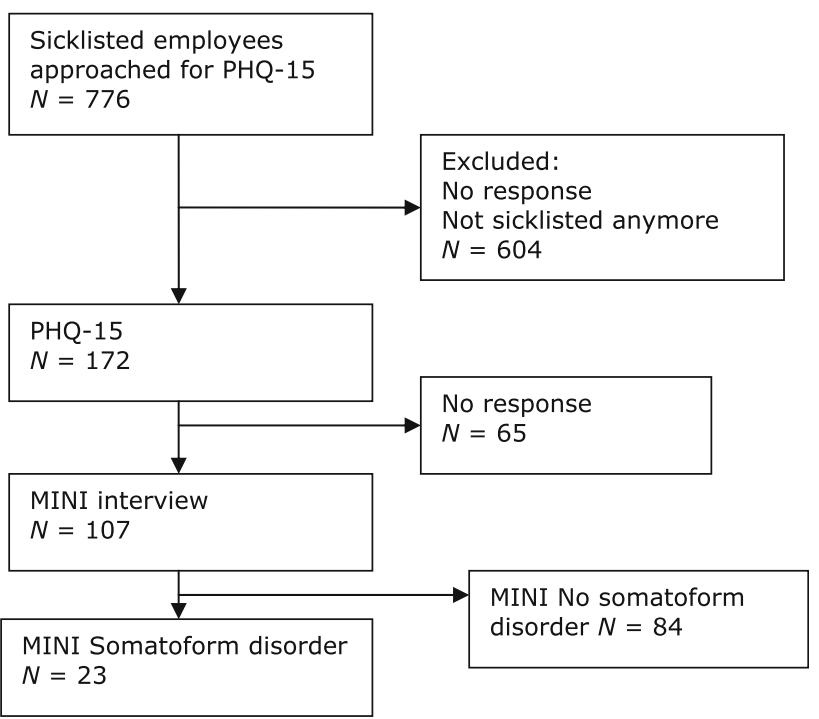

Fig. 1 Report of the number of participants during the course of our study

Table 1 Disorder classifications and PHQ-15 scores

Somatoform disorder according to MINI $(N=23)$

MINI classification

Pain disorder

Pain disorder RSI

Undif. cardiac pain

Undif. soma. dis. fibromyalgia

Undif. chronic fatigue

Undifferentiated IBS

Undif. somatoform

Conversion disorder

Somatoform disorder

Undif undifferentiated, IBS irritable bowl syndrome, $R S I$ repetitive strain injury, soma. dis. somatoform disorder

"somatoform disorder group"); this is a prevalence of $21.5 \%$. Amongst the somatoform disorders, pain disorders were the most prevalent with a total prevalence of $47.8 \%$ $(N=11)$. The next most frequent somatoform disorder was chronic fatigue, $21.7 \%(N=5)$, followed by IBS $(N=2,8.7 \%)$ and undifferentiated somatoform disorder $(N=3,13.0 \%)$. Conversion $(N=1)$ and somatoform disorder $(N=1)$ were scarce.

\section{Demographic Characteristics}

None of the demographic characteristics showed a significant difference between the somatoform disorder group and the no-somatoform disorder group. The total sample existed of 53 male $(49.5 \%)$ participants. The mean age was $47.9(\mathrm{SD}=9.8) .13 .1 \%(N=14)$ of the participants stated to be single, $74.8 \%(N=80)$ were living together or were married and $12.1 \%(N=13)$ said to be divorced or be widow/widower. A total of $31.8 \%(N=34)$ participants finished an education at low level, $38.3 \%(N=41)$ at middle high level and $29.9 \%(N=32)$ at high level.

Before sick leave, participants on average worked 4.2 $(\mathrm{SD}=1.2)$ days a week, which corresponds to 30.3 $(\mathrm{SD}=11.2)$ hours a week. Almost all participants $(98.1 \%)$ stated to be in paid employment. Only $12.1 \%$ of the participants fulfilled an executive function and $64.5 \%$ declared to be wage earner. All employees included in the study were sicklisted during the study.

\section{Mean Scores on PHQ-15}

The mean PHQ-15 score in the total study sample was 8.3 $(\mathrm{SD}=4.6$; range $=1-22)$. The difference between the means in the somatoform disorder group (mean $=10.1$, $\mathrm{SD}=5.5$, range $=1-22$ ) and the no somatoform disorder group $(7.8 ; \mathrm{SD}=4.1 ;$ range $=1-19)$ was significant $(P=0.030)$, confirming the construct validity of the PHQ15 (Table 1).

\section{Validation Scores}

Table 2 shows the sensitivity, the specificity, and the predictive values for both positive and negative test results (positive predictive value (PPV), negative predictive value $(\mathrm{NPV})$ ) and the efficiency. These validation scores were

Table 2 Validation scores of the PHQ-15

\begin{tabular}{lllllll}
\hline Frequency & $\begin{array}{l}\text { Score } \geq 6 \\
N=74(79.2 \%)\end{array}$ & $\begin{array}{l}\text { Score } \geq 7 \\
(\%) 61(65.3 \%)\end{array}$ & $\begin{array}{l}\text { Score } \geq 8 \\
N=52(55.6 \%) \\
(\%)\end{array}$ & $\begin{array}{l}\text { Score } \geq 9=45(48.2 \%) \\
(\%)\end{array}$ & $\begin{array}{l}\text { Score } \geq 10 \\
N=37(39.6 \%) \\
(\%)\end{array}$ & $\begin{array}{l}\text { Score } \geq 11 \\
N=27(28.9 \%) \\
(\%)\end{array}$ \\
\hline Sensitivity & 82.6 & 69.6 & 60.9 & 56.5 & 52.2 & 39.1 \\
Specificity & 34.5 & 46.4 & 54.8 & 61.9 & 70.2 & 78.6 \\
NPV & 25.7 & 26.2 & 26.9 & 28.9 & 32.4 & 33.3 \\
PPV & 87.9 & 84.8 & 83.6 & 83.9 & 84.3 & 82.5 \\
Efficiency & 30.8 & 43.0 & 51.4 & 57.9 & 65.4 & 74.8 \\
\hline
\end{tabular}

$N P V$ negative predictive value, $P P V$ positive predictive value 
calculated for the total score on the PHQ-15 that ranged from 0 to 30. A range of PHQ-15 scores was considered in order to establish the optimal cut point.

Sensitivity was the highest $(82.6 \%)$ at a cut point of 6 . However, at cut point 6 the specificity of $34.5 \%$ was unacceptably low. Maximum specificity of $78.6 \%$ was found at cut point of 11 , but then sensitivity was $39.1 \%$, NPV was $33.3 \%$ and PPV was $82.5 \%$. Efficiency was $74.8 \%$, the highest value for all possible cut points. A cut point of 9 resulted in sensitivity of $56.5 \%$ and specificity of $61.9 \%$, NPV of $28.9 \%$, and PPV of $83.9 \%$. At cut point 9 , efficiency was $57.9 \%$.

\section{ROC Analysis}

Figure 2 shows the ROC for the PHQ-15 versus the MINI as golden standard.

Table 3 shows the outcomes of the ROC analysis in terms of AUC, standard error (SE) and confidence interval (CI).

The AUC of the PHQ-15 versus the MINI was 0.63 $(\mathrm{SE}=0.07 ; 95 \%$ CI: $0.50-0.76)$.

\section{Discussion}

\section{Prevalence}

In this study, in a sicklisted population, 23 out of 107 sicklisted employees were classified with a somatoform disorder according to the MINI interview, which is a

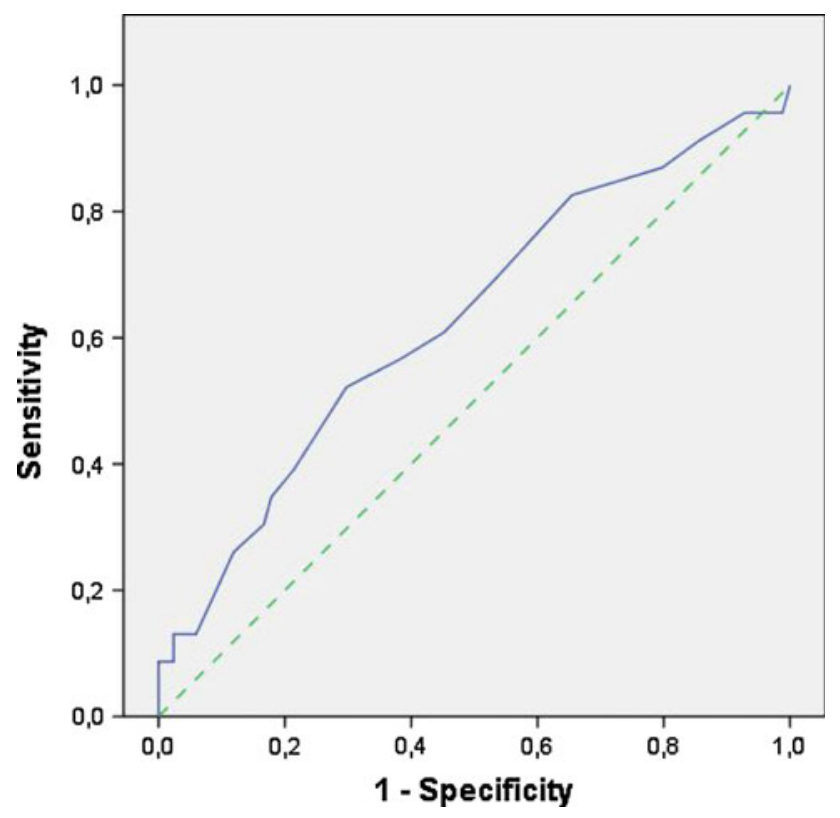

Fig. 2 ROC curve for PHQ-15 versus M.I.N.I. ROC-curve with the dotted line is the reference line
Table 3 Outcomes of the ROC analysis for PHQ-15

\begin{tabular}{llllll}
\hline AUC & $\begin{array}{l}\text { Std. error } \\
\text { (a.) }\end{array}$ & $\begin{array}{l}\text { Asymptotic } \\
\text { sig. (b.) }\end{array}$ & \multicolumn{2}{c}{ Asymptotic 95\%CI } \\
\cline { 5 - 6 } & & & $\begin{array}{l}\text { Upper } \\
\text { bound }\end{array}$ & $\begin{array}{l}\text { Lower } \\
\text { bound }\end{array}$ \\
\hline PHQ-15 & 0.63 & 0.07 & 0.06 & 0.50 & 0.76
\end{tabular}

$A U C$ area under the curve

CI confidence interval

prevalence of $21.5 \%$. This prevalence is higher than the prevalence found by Hoedeman et al. [3], in a comparable sick listed population, performed by questionnaire alone. The explanation may be that Hoedeman et al. chose a cut point of 15 or more on the PHQ-15; in view of the present findings, using such a high cut point results in missing a substantial number of cases of somatoform disorders in the $\mathrm{OH}$ setting. Given the findings from the MINI and given comparable PHQ-15 mean scores (9.8, SD 5.4) in Hoedeman et al.'s study and ours (10.1, SD 5.5), a cut point of 15 may be unnecessarily high to detect somatoform disorders by means of the PHQ-15 in the $\mathrm{OH}$ setting.

In the primary care population, Ravestijn et al. found a mean on the PHQ-15 of 6.1 (SD 5.3). The difference between primary care patients and sick-listed patients may play a role in the lower mean scores in the Ravestijn et al. study; not all primary care patients with MUS will have such a degree of job disfunction that they are sicklisted. So the sample in this study in the $\mathrm{OH}$ setting probably suffers more serious disfunctioning than the primary care sample of Ravesteijn et al. [9].

The MINI classifications show that the most prevalent somatoform disorders in this sicklisted population are pain disorder $(48 \%)$ and chronic fatigue (22\%). This finding confirms findings of Nimnuan et al. [12] who established that pain and fatigue were MUS that could be found in many somatoform disorders. Furthermore, in this study, Irritable Bowel Syndrome (IBS) occurred but less frequently. This confirms findings of Fink et al. [13] who also found IBS to be one of the three most frequent MUS in a primary care population. Apparently, these are the most relevant symptoms in MUS in the sicklisted population as well, although prevalence rates for IBS are lower here than in Fink's study. The explanation may be that although pain and fatigue are strongly associated with disfunctioning at work and thus with being on sick leave, IBS may not be so disabling in employees in general that it leads to sick leave.

Validity of the PHQ-15

The results presented in this article show that the PHQ-15 can be a highly sensitive screener if a cut point of 6 is used, 
with a sensitivity of $82,6 \%$. Maximum specificity (78.6\%), and efficiency $(74.8 \%$ ) were found at cut point 11 , which is moderate for both measures. However, at a cut point of 11 sensitivity is unacceptably low. If the optimal balance between sensitivity and specificity is sought, a cut point of 9 yields sensitivity of $56.5 \%$ and specificity of $61.9 \%$, and an AUC of 0.63 . The validity of the PHQ-15 as a screening instrument for assessing somatoform disorders in the $\mathrm{OH}$ setting can thus be considered low [14] to moderate [15].

As far as we know, there is no screener for somatoform disorders with more than moderate validity. Our data show that sick-listed employees without somatoform disorders have scores on the PHQ-15 of at least 5, which Kroenke et al. consider a mild severity of somatic symptoms [11]; however, in this study the mean score on the PHQ-15 is significantly smaller than in the group classified by the MINI as having a somatoform disorder. MUS is associated with poor prognosis, possibly partly due to low recognition by OHPs and lack of better screeners for somatoform disorders. Moreover, the number of MUS is associated with poor prognosis, high medical consumption, and longer sickness absence [3]. Van der Feltz-Cornelis et al. [16] reported that adequate recognition and treatment can speed up Return To Work (RTW) considerably. Therefore, the use of the PHQ-15 as a screener to detect somatoform disorders may still be of high clinical relevance.

The cut point of 9 is higher than reported in the primary care study of Ravestijn et al. which studied the validity of the PHQ-15 using the SCID-1 as golden standard instead of the MINI. The study sample of Ravestijn et al. existed of a high risk primary care population with patients known to suffer from MUS, frequent attenders and patients with mental health problems. They reported a sensitivity of $78 \%$ and specificity of $71 \%$ at the optimal cut point of 6 [9]. The difference between primary care patients and sick-listed patients may play a role in the lower cut point in the Ravestijn et al. study. Probably, the PHQ-15 can be used with a lower cut point in patients in primary care, whereas in the $\mathrm{OH}$ setting, in a sample of sicklisted employees with a longer duration of sickness absence and thereby a negative selection of employees with symptoms (most sicklisted employees return to work with a shorter duration of sickness absence than 2 weeks), as shown by our data, the optimal cut point is 9 .

\section{Nonresponse Analysis}

Our findings show no relationship between demographic variables and scores on the PHQ-15 between responders and non-responders. It can therefore be stated that it does not matter, for instance, which gender the participant has. Participants' scores on either the MINI or PHQ-15 will not be influenced by such factors. These results show that there is no evidence for selectivity within our study sample in terms of demographic characteristics and severity of physical symptoms.

\section{Implications for Clinical Practice}

The findings suggest that the PHQ-15 may be used as a screener in the $\mathrm{OH}$ setting, in order to alert the OHP of the possibility of somatoform disorders. Due to the rather low efficiency of the instrument, it may be best to apply the screener in high risk groups. The general literature suggests that patients in the primary care setting with more than 4-6 symptoms were more often disabled [17]. Frequent doctor visits were also associated with disability. Furthermore, research in the $\mathrm{OH}$ setting showed that such high risk groups, amongst disabled and sick-listed employees, might be those with many MUS [18], with high medical consumption [17, 18], who report to be severely disabled [19]. Also, for sick-listed employees with depression or anxiety disorder it was shown [21] that higher age [20] as well as negative expectation of the employee regarding duration of sickness absence contributed to longer duration of sickness absence. Maybe high age and such expectations of the employee regarding Return To Work(RTW) should also be an indication to screen for MUS with the PHQ15. Although the PHQ-15 might not be helpful enough as a stand-alone screener, it could have promising possibilities when used in such high risk groups. The Multidisciplinary guideline for MUS and somatoform disorder [22] or the Dutch multidisciplinairy guideline [7] might be useful to provide the OHP with evidence based treatment options.

Occupational rehabilitation for employees with somatoform disorders could be improved by applying management rules for management and communication. Evidence [23] is indirect as effectivity was shown in primary care, after establishing the diagnosis by psychiatric screening; and further investigation of the effectivity in the sick-listed population is needed, but these interventions showed in primary care improvement of functioning and reduction of medical consumption. If the process of RTW in employees with somatoform disorders is hampered, referral for cognitive behavioral therapy (CBT) or multidisciplinary treatment with graded activity and CBT is indicated. These treatments have shown to be effective for the outcome of functioning [24].

Implications for Research

Further research is needed to validate the PHQ-15 in highrisk groups for somatoform disorder in sicklisted employees. Furthermore, the low to moderate efficiency of the PHQ-15 may have to do with the possibility of comorbid depressive and anxiety disorder in somatoform disorders. 
This comorbidity is highly prevalent [25] and has a negative influence on the course of illness as well as on treatment outcome [26, 27]. In this study, no comorbid conditions were found in the MINI interview. However, more research is needed to explore this possibility, and its implications for the validity of the PHQ-15 for this patient group.

\section{Strengths and Limitations of the Study}

A return rate for a mail questionnaire of $22.1 \%$ is commonly found but it is a small percentage. Another limitation is that not all participants who filled out the questionnaire also consented to the MINI interview; $62 \%$ did $(N=107)$. Nonresponse was probably due to the eligible persons being approached twice, once for the mail questionnaire, and a second time for the MINI interview. However, a non-response analysis did not show significant differences between responders and non-responders at least in terms of demographic characteristics. The reported health reasons for being sick-listed in this study are unknown. In a comparable population with a random sample of Dutch employees being sick-listed between 3 weeks and 2 years [3] the OHP diagnoses were for $40 \%$ mental, $30 \%$ musculoskeletal and for $30 \%$ other disorders.

The application of the MINI interview to diagnose somatoform disorders as golden standard is a strength of this study. For example, in another validation study that reported high internal reliability, convergent validity and discriminant validity for the PHQ-15, the PHQ-15 was compared with the outcomes on the 20-item Short-Form General Health Survey (SF-20) as golden standard [11]. We compared the PHQ-15 to the more valid MINI. Another strength of the study is that sicklisted employees were approached by questionnaire, thus eliminating selection bias by the OHP. Finally, our study is the first to validate the PHQ-15 in the OH Setting.

\section{Conclusion}

This article contributes to the question whether or not the PHQ-15 can be used as a screening instrument to detect somatoform disorders in the $\mathrm{OH}$ setting. The PHQ-15 is a questionnaire with moderate validity, i.e. reasonable sensitivity but limited efficiency. Due to its sensitivity, it can be used as a screener for somatoform disorders in the $\mathrm{OH}$ setting. This may be particularly promising in high-risk groups. In view of the high prevalence of MUS and somatoform disorders in the $\mathrm{OH}$ setting, and in view of the findings that no known screener for MUS has been established for more than moderate validity [9], the PHQ-15 and the establishment of its cut point for a sicklisted population is therefore of significant clinical relevance. However, further research is needed.

Acknowledgments The authors of this article would like to thank Melanie de Ruiter for performing the MINI interviews.

Open Access This article is distributed under the terms of the Creative Commons Attribution Noncommercial License which permits any noncommercial use, distribution, and reproduction in any medium, provided the original author(s) and source are credited.

\section{References}

1. Shima S, Satoh E. Somatoform disorders in the workplace in Japan. Int Rev Psychiatr. 2006;18:35-40.

2. de Waal MW, Arnold IA, Eekhof JA, van Hemert AM. Somatoform disorders in general practice: prevalence, functional impairment and comorbidity with anxiety and depressive disorders. Br J Psychiatry. 2004;184:470-6.

3. Hoedeman R, Krol B, Blankenstein N, Koopmans PC, Groothoff JW. Severe MUPS in a sick-listed population: a cross-sectional study on prevalence, recognition, psychiatric co-morbidity and impairment. BMC Public Health. 2009;9:440.

4. van der Feltz-Cornelis CM, Meeuwissen JA, de Jong FJ, Hoedeman R, Elfeddali I. Randomised controlled trial of a psychiatric consultation model for treatment of common mental disorder in the occupational health setting. BMC Health Serv Res. 2007;7:29.

5. Leikness KA, Finset A, Moum T, Sandanger I. Current somatoform disorders in Norway: prevalence, risk factors and comorbidity with anxiety, depression and musculoskeletal disorders. Soc Psychiatry Psychiatr Epidemiol. 2007;42:698-710.

6. Mergl R, Seidscheck I, Allgaier AK, Moller HJ, Hegerl U, Henkel V. Depressive, anxiety, and somatoform disorders in primary care: prevalence and recognition. Depression and Anxiety. 2007;24:185-95.

7. van der Feltz-Cornelis CM, Swinkels JA, Blankenstein AH, Hoedeman R, Keuter EJW. The Dutch multidisciplinairy guideline entitled 'Medically unexplained physical symptoms and somatoform disorder'. Ned Tijdschr Geneeskd. 2011;155:A1244.

8. Kroenke K, Spitzer RL, Williams JB. The PHQ-15: validity of a new measure for evaluating the severity of somatic symptoms. Psychosom Med. 2002;64:258-66.

9. van Ravesteijn HJ, Wittkampf K, Lucassen P, van de Lisdonk E, van den Hoogen $\mathrm{H}$, van Weert WH, Huijser J, Schene A, van Weel C, Speckens A. Detecting somatoform disorders in primary care with the PHQ-15. Ann Fam Med. 2009;7:232-238.

10. Sheehan DV, Lecrubier Y, Sheehan KH, Amorim P, Janavs J, Weiller E, Hergueta T, Baker R, Dunbar GC. The Mini-International Neuropsychiatric Interview (M.I.N.I.): the development and validation of a structured diagnostic psychiatric interview for DSM-IV and ICD-10. J Clin Psychiatry. 1998;59(Suppl 20): 22-33.

11. Kroenke K, Spitzer RL, Williams JB, Löwe B. The patient health questionnaire somatic, anxiety, and depressive symptom scales: a systematic review. Gen Hosp Psychiatry. 2010;32(4):345-59.

12. Nimnuan C, Rabe-Hesketh S, Wessely S, Hotopf M. How many functional somatic syndromes? J Psychosom Res. 2001;51(4): 549-57.

13. Fink P, Toft T, Hansen MS, Ørnbøl E, Olesen F. Symptoms and syndromes of bodily distress: an exploratory study of 978 internal medical, neurological, and primary care patients. Psychosom Med. 2007;69(1):30-9. 
14. Fischer JE, Bachmann LM, Jaeschke R. A readers' guide to the interpretation of diagnostic test properties: clinical example of sepsis. Intensive Care Med. 2003;29:1043-51.

15. Jones CM, Athanasiou T. Summary receiver operating characteristic curve analysis techniques in the evaluation of diagnostic tests. Ann Thorac Med. 2005;79:16-2016.

16. van der Feltz-Cornelis CM, Hoedeman R, Drewesen HW, Meeuwissen JA, de Jong FJ, Van der Laan NC, Adèr HJ. Faster return to work after psychiatric consultation for sicklisted employees with common mental disorders compared to care as usual. A randomized clinical trial. Neuropsychiatr Dis Treat. 2010;6:375-85.

17. Escobar JI, Waitzkin H, Silver RC, Gara M, Holman A. Abridged somatization: a study in primary care. Psychosom Med. 1998; 60(4):466-72.

18. Jackson JL, Passamonti M. The outcomes among patients presenting in primary care with a physical symptom at 5 years. J Gen Intern Med. 2005;20:1032-7.

19. Kroenke K, Spitzer RL, DeGruy FV, Hahn SR, Linzer M, Williams JB, Brody D, Davies M. Multisomatoform disorder. An alternative to undifferentiated somatoform disorder for the somatizing patient in primary care. Arch Gen Psychiatry. 1997;54: $352-8$.

20. Hoedeman R, Blankenstein AH, Krol B, Koopmans PC, Groothoff JW. The contribution of high levels of somatic symptom severity to the duration of sickness absence, disability and discharge. J Occup Rehabil. 2010;20:264-73.
21. Nieuwenhuijsen K, Verbeek JH, De Boer AG, Blonk RW, van Dijk FJ. Predicting the duration of sickness absence for patients with common mental disorders in occupational health care. Scand J Work Environ Health. 2006;32:67-74.

22. Multidiciplinary guideline. Somatic insufficient explained physical symptoms and somatroform disorders. Trimbos Institute. 2010.

23. Hoedeman R, Blankenstein AH, van der Feltz-Cornelis CM, Krol B, Stewart RE, Groothoff JW. Consultation letters for medically unexplained physical symptoms. Cochrane Database Syst Rev. 2010;8:CD006524.

24. Henningsen P, Zipfel S, Herzog W. Management of functional somatic symptoms. Lancet. 2007;369:946-55.

25. van der Feltz-Cornelis CM, van Balkom AJ. The concept of comorbidity in somatoform disorder-a DSM-V alternative for the DSM-IV classification of somatoform disorder. J Psychosom Res. 2010;68(1):97-9.

26. Huijbregts KM, van der Feltz-Cornelis CM, van Marwijk HW, de Jong FJ, van der Windt DA, Beekman AT. Negative association of concomitant physical symptoms with the course of major depressive disorder: a systematic review. J Psychosom Res. 2010; 68(6):511-9.

27. Huijbregts KML, van Marwijk HWJ, de Jong FJ, Schreuders B, Beekman ATF, van der Feltz-Cornelis CM. Adverse effects of multiple physical symptoms on the course of depressive and anxiety symptoms in primary care. Psychother Psychosomatics. 2010;79:389-91. 